\title{
Staff Perceptions of Organizational Patient Safety Climate and Quality Outcomes: An Examination of Outpatient Medical Offices
}

\author{
Jason P. Richter ${ }^{1}$, Brad Beauvais ${ }^{2 *}$, Lynn Downs ${ }^{3}$, Matthew Calvert ${ }^{4}$, Fernando Najera ${ }^{5}$, Steven Wentz ${ }^{6}$, Bob \\ Wolfe ${ }^{7}$, and Forest Kim ${ }^{8}$
}

\author{
1 Administrator, Aviano Medical Clinic, Aviano Air Base, Aviano, Italy; jason.p.richter4.mil@mail.mil, \\ 2* Assistant Professor, Texas State University, San Antonio, TX, bmb230@txstate.edu; Tel: (210) 627-1078 \\ 3 Assistant Professor, University of Incarnate Word, San Antonio, TX, 1downs@uiwtx.edu, \\ 4 Regional Practice Manager, Health, Safety and Work-Life, USCG Portsmouth, mattclvrt4@gmail.com \\ 5 Deputy Director of Clinical Operations, Portsmouth, Virginia, orange.najera@gmail.com \\ 6 Student at the US Army-Baylor MHA Program, gostevewentz@yahoo.com \\ 7 HM Rating Assignment Office at PERS, Jacksonville, FL, wolfe613@yahoo.com \\ 8 Program Director, Baylor University, Waco, TX, Forest Kim@baylor.edu
}

\begin{abstract}
As many as $20-25 \%$ of the population experiences harm in outpatient settings, yet these locations are underrepresented in the literature compared to hospitals. We examined results from the Medical Office Survey on Patient Safety Culture designed by the Agency for Healthcare Research and Quality. The survey administered in 2012 gathered perceptions from 23,679 individuals in 934 unique medical offices. We examined associations of organizational patient safety climate composites on frequency of safety and quality issues, overall quality score, and safety rating. We found organizational patient safety composites are all positively and significantly associated with a higher overall quality score and patient safety rating, and fewer safety and quality issues. Office processes and standardization appeared to have the most consistent influence on perceived quality outcomes. Our results indicate it may be advantageous for medical offices to improve on the factors that contribute to positive safety climate.
\end{abstract}

Keywords: errors, culture, quality improvement, patient safety, management, clinics, outpatient

\section{Introduction}

Creating a patient safety climate among hospital staff and providers has proven to be extremely challenging due to conflicting demands to control costs and increase efficiency along with a traditional professional hierarchy [1]. That challenging environment makes it important to understand which organizational elements of safety climate have the greatest impact on safety climate and outcomes. Organizations with a positive safety climate are characterized by trustworthy communications, shared perceptions of safety, and effectiveness of preventive measures [2]. Richter, McAlearney, and Pennell identified that error feedback and organizational learning were the most significant predictors of error reporting [3]. Meanwhile, Liu, Liu, Wang, Zhang, and Wang found that specific elements of organizational safety culture, such as communication openness and perceived penalties from error reporting, may have a stronger influence than other organizational factors on patient outcomes in inpatient settings [4]. 


\section{New Contribution}

As many as 20-25 percent of the population experiences harm in the primary care setting [5]. While 900 million visits occur in medical offices, compared to 35 million hospital discharges, only $10 \%$ of patient safety studies have been performed in outpatient settings, with most focusing on medication errors [6]. That research indicates that safety issues in the outpatient setting may differ from those in the inpatient setting. Outpatient visits are shorter than inpatient stays, are fragmented, some focus on prevention instead of healing a condition, and the risk for infection during the visit is much less. However, differences do not indicate that patient safety in outpatient settings is unimportant, as it is estimated that 30 percent of safety issues originate prior to hospital admission, with many of those issues related to primary care [7].

Although there exists growing literature on the relationship between patient safety climate and quality and safety performance in inpatient settings, there has been limited research on the elements of patient safety climate and outcomes in outpatient care [8]. One example is a survey of small family practices with teams of 10 or fewer professionals that found a positive correlation between patient safety climate and reporting of adverse events in primary care [9]. Our research will add to previous studies by testing the association of specific elements of patient safety climate with overall safety perceptions and ratings in outpatient care settings.

\section{Conceptual Model and Hypotheses}

The Vogus, Sutcliffe, and Weick model of enabling, enacting, and elaborating activities that impact safety outcomes, forms the conceptual framework for our study [10]. This model provides a comprehensive and integrative framework of how patient safety is produced and sustained through safety culture. Enabling consists of leader actions that draw attention to safety. Enacting are actions by frontline staff to highlight threats to safety and mitigate those threats. Elaborating involves learning actions that reflect on past safety outcomes and use feedback to improve. If these constructs are well established then optimal safety outcomes can be reasonably expected.

We impose the lens of enabling, enacting, and elaborating a safety culture onto the Medical Office Survey on Patient Safety Culture (MOSOPS). Owner support for safety is reflective of an enabling activity. Meanwhile, info exchange, teamwork, work pressure and pace, staff training, office processes and standardization, communication openness, patient care tracking are enacting activities. Communication about errors and organizational learning are examples of elaborating activities. In our study, the safety outcomes are perceived outcomes, ratings, and frequency of safety issues. This conceptual model and research that shows that patient safety climate has a direct relationship with patient outcomes leads us to our three hypotheses [11]:

H1: Higher staff perceptions of organizational elements of patient safety climate in outpatient settings will be associated with higher overall perceptions of safety.

H2: Higher staff perceptions of organizational elements of patient safety climate in outpatient settings will be associated with higher overall ratings of safety. 
H3: Higher staff perceptions of organizational elements of patient safety climate in outpatient settings will be associated with a lower frequency of safety issues.

\section{Materials and Methods}

Data and Sample

We conducted a cross-sectional evaluation with data obtained from the AHRQ database, collected through the MOSOPS, and de-identified by Westat. A link to the survey and its composites is provided here: https://www.ahrq.gov/professionals/qualitypatient-safety/patientsafetyculture/medical-office/index.html

The results we received originated from 23,679 individuals in 934 unique medical offices that submitted data to AHRQ; no sampling was conducted. The response rate for each medical office ranged from 7-100 percent, with an average of 71 percent. Respondents included physicians, physician extenders (i.e. physician assistants, nurse practitioners, etc.), administrative staff, management, nurses (RNs, LVNs, and LPNs), and other medical support staff. Our unit of analysis was the organization because we were interested in the perceptions and outcomes at an organizational level, specifically the outpatient medical office. The data used within our study reflect responses to the MOSOPS received by AHRQ in the year 2012.

The MOSOPS had a pilot run, to include 4,200 surveys across 202 medical offices, to determine the reliability of the questions to their composites. Each composite consisted of 3-9 related questions. AHRQ calculated Cronbach alpha scores for each composite that ranged from $0.75-0.90$, demonstrating a high level of construct validity for each.

\section{Measures}

Our study concentrated on the following three dependent variables (DVs) that depict summary assessments of safety and quality: overall perceptions of patient safety and quality, frequency of patient safety and quality issues, and overall quality and patient safety rating. These questions focused more on an overall assessment of quality and safety and used terminology such as "overall" in the AHRQ composite title. The "frequency of patient safety and quality issues" composite was used as a DV because the questions more closely reflected safety errors or issues that directly impacted the patient. The overall patient safety and quality variable was based on four questions related to the number of mistakes and preventing them. For example, one question read, "Our office processes are good at preventing mistakes that could affect patients". The overall quality rating variable was a composite of five questions, as to how the medical office care quality was patientcentered, effective, timely, efficient, and equitable. The frequency of patient safety and quality issues variable is a composite of nine questions related to the frequency of issues with the following: access to care, patient identification, medical records, medical equipment, medication, and diagnostic tests. We used percent positive scores for each variable. To get this, we first determined the percent of responses for each medical office that responded to a question in a positive manner (i.e. agree or strongly agree; most of the time or always; very good or excellent; problems once or twice in the past 12 months or no problems in the past 12 months). For the frequency of safety and quality issues variable, 
we considered a positive response to be fewer issues such as "several times in the past 12 months" or "not in the past 12 months." Responses not considered positive were "daily" "weekly" and "monthly." Then we averaged the scores for the questions within each composite, to make the composite score used for the variable. Percent positive scores could theoretically range from 0 to 100, with a higher percent positive score indicating better quality and safety. The approach to using percent positive scores is a widely accepted approach for data from the similar hospital SOPS survey [3,17].

In order to determine which perceived elements of organizational patient safety climate impact overall perceived ratings, perceptions, and issues, we looked at ten primary independent variables (IVs). Similar to our DVs, these variables reflect data obtained from MOSOPS survey questionnaires of medical office staff about their perceptions of patient safety climate. The IVs included in our empirical model are: information exchange with other settings, teamwork, work pressure and pace, staff training, office processes and standardization, communication openness, patient safety tracking, communication about error, leadership support for patient safety, and organizational learning. Similar to the DVs, the variables are composites of 3-5 questions that reflect percent positive scores.

Our control variables were limited by the variables contained in our data set. We included variables on size, ownership type, and location. This is similar to other related studies that controlled for hospital size, teaching status, and ownership type [12]; type of practice, population size, and location [13]; hospital size, type, ownership, and staffing levels on perceptions of patient safety [14]. Furthermore, in the outpatient setting, owner type has shown a significant impact on safety climate [15]. The MOSOPS survey contains information on four control variables that we used: region (New England/Mid-Atlantic, South Atlantic, East Central, West North Central, West South Central, or Mountain/Pacific), owner type (provider/physician, university or academic institution, hospital or health system, community health center, or government-owned), practice type (single or multispecialty), and number of providers working ( 1 per week, 2 per week, 3 per week, 4-9 per week, 10-13 per week, 14-19 per week, or 20+ per week).

\section{Analysis}

To account for the difference in proportion of respondents for each medical office, we analyzed the data using weighted least squares regression, using response rate as the weight. The main advantage weighted least squares has over a standard linear regression is its ability to handle situations in which the data points are of varying quality [16]. Because some medical offices had low response rates and some had high, we wanted to account for that. We also conducted a sensitivity analysis where we removed all 133 medical offices that had a lower than 50 percent response rate, and found no change in levels of statistical significance and very minimal change in beta coefficients. Because there were high correlations between the independent variables, we ran a unique linear regression model for each IV and DV, while including the four control variables in each model. In each case, variance inflation factor $(\mathrm{VIF})<10$ failed to present any indication of multicollinearity, although the relatively high correlations among variables suggested we 
run the models separately. Running the models separately is consistent with published literature on HSOPS data [17-18]. Similarly, we assessed the partial r-square for each primary IV on each DV to determine the degree to which the variation in each patient safety outcome variable is explained by each specific composite of organizational safety climate.

Since the IVs and DVs were obtained from the same survey instrument, we conducted Harman's single factor test and a confirmatory factor analysis which is consistent with the method used by other studies. We assessed common method bias using Harman's Single Factor test. The common method variance (CMV) was 57\%, below the $70 \%$ level determined to be problematic [19]. All statistical analysis of our data was accomplished utilizing IBM Statistical Package for the Social Sciences (SPSS) software version 24 .

\section{Results}

Table 1 illustrates the types of medical offices that reported MOSOPS survey results. The highest proportion of medical offices in our sample reside in the East Central region (35 percent), which includes Illinois, Indiana, Michigan, Ohio, and Wisconsin. The medical offices were predominantly single specialty practices (69 percent), owned by a hospital or health system (73 percent), with an average of 4-9 providers working per week (41 percent).

Table 1. Characteristics of Medical Offices

\begin{tabular}{lr}
\hline & $\%$ \\
\hline Region: & \\
New England/Mid-Atlantic & 13.7 \\
South Atlantic & 27.6 \\
East North Central & 34.9 \\
West North Central & 12.1 \\
West South Central & 6.3 \\
Mountain/Pacific & 5.4 \\
Owner Type: & \\
Provider/Physician & 10.3 \\
University & 6.1 \\
Hospital or Health System & 73.0 \\
Community Health Center & 6.6 \\
Government & 4.0 \\
Number of Providers per Week: & \\
1 & 3.7 \\
2 & 12.0 \\
3 & 11.9 \\
$4-9$ & 41.0 \\
$10-13$ & 9.5 \\
$14-19$ & 6.9
\end{tabular}


$20+$

Practice Type

\begin{tabular}{rr} 
Single Specialty & 68.6 \\
Multi-Specialty & 31.4 \\
\hline $\mathrm{N}=934$ medical offices &
\end{tabular}

$\mathrm{N}=934$ medical offices

Table 2 indicates the percent positive responses for each of our IVs and DVs. Overall rating was the lowest scoring DV (65 percent positive), with frequency of issues scoring highest (81 percent). For the IVs, work pressure and pace was the lowest scoring (47 percent) and teamwork the highest (84 percent).

Table 2. Percent Positive Response by Composite

\begin{tabular}{lrr}
\hline & Mean & SD \\
\hline DV: & & \\
Overall perceptions of patient safety \& quality & 76.2 & 12.6 \\
Frequency of patient safety and quality issues & 80.7 & 9.1 \\
Overall quality \& patient safety rating & 65.4 & 13.7 \\
IV: & & \\
Info Exchange & 77.4 & 12.8 \\
Teamwork & 84.2 & 10.6 \\
Work pressure and pace & 46.7 & 16.8 \\
Staff training & 72.8 & 13.3 \\
Office processes \& standardization & 63.6 & 14.7 \\
Communication openness & 64.6 & 14.1 \\
Patient care tracking & 82.0 & 10.3 \\
Communication about errors & 66.8 & 12.7 \\
Owner support for safety & 66.7 & 13.5 \\
Organizational learning & 76.9 & 12.3 \\
\hline N=934 medical offices & &
\end{tabular}

We found support for the Vogus et al. model in that enabling, enacting, and elaborating activities all affect quality outcomes. Our results are consistent with our hypotheses that there is a positive association between organizational elements of patient safety climate within outpatient facilities and overall patient safety perceptions, overall quality ratings, and a negative association with frequency of safety and quality issues. The adjusted R-squared values ranged from 0.24 to 0.62 in our models.

\section{Overall Perceptions of Patient Safety and Quality}

With regard to our first DV, overall perception of patient safety and quality, each model and IV was found to be statistically significant with $p<.001$. The adjusted Rsquared ranged from 0.17 to 0.57 for the different models. Teamwork $(\beta=0.77)$ and organizational learning $(\beta=0.75)$ had the largest coefficients, while office processes and 
standardization (partial $\mathrm{R}^{2}=0.57$ ) and organizational learning (partial $\mathrm{R}^{2}=0.54$ ) explained the most variation in overall perceptions of patient safety and quality.

Frequency of Patient Safety and Quality Issues

Evaluation of our IVs in relation to our second DV, frequency of patient safety and quality issues, again indicated that each IV and each model was significantly associated to the DV. Each analysis had significant relationships at the $p<.001$ level. The adjusted Rsquared ranged from 0.22 to 0.47 for the different models. Information exchange $(\beta=0.45)$ and patient care tracking $(\beta=0.44)$ had the largest coefficients, while information exchange (partial $\mathrm{R}^{2}=0.42$ ) and office processes and standardization (partial $\mathrm{R}^{2}=0.25$ ) explained the most variation in overall perceptions of patient safety and quality.

\section{Overall Quality and Patient Safety Rating}

Our final analysis focused on overall rating on quality and patient safety. Each model and IV was statistically significant at a level of $p<.001$, while adjusted R-squared ranged from 0.24 to 0.48 . Teamwork $(\beta=0.74)$, communication about errors $(\beta=0.65)$, and organizational learning $(\beta=0.64)$ had the largest coefficients. Communication about errors (partial $R^{2}=0.40$ ) and office processes and standardization (partial $R^{2}=0.39$ ) explained the most variation in overall perceptions of patient safety and quality.

Table 3 summarizes the partial R-square values from all analyses. When looked upon in totality for the partial R-squares, office processes and standardization had the most consistently high relationship with the three outcome variables, with organizational learning next. When examining beta coefficients, teamwork was highest with organizational learning next.

Table 3. Regression Analyses

\begin{tabular}{|c|c|c|c|c|c|c|}
\hline & \multicolumn{2}{|c|}{$\begin{array}{c}\text { Overall } \\
\text { Perceptions }\end{array}$} & \multicolumn{2}{|c|}{$\begin{array}{c}\text { Frequency of } \\
\text { Issues }\end{array}$} & \multicolumn{2}{|c|}{ Overall Rating } \\
\hline & $\beta$ & Partial R2 & $\beta$ & Partial R ${ }^{2}$ & $\beta$ & Partial $R^{2}$ \\
\hline Info Exchange & 0.40 & 0.17 & 0.45 & 0.42 & 0.36 & 0.12 \\
\hline Teamwork & 0.77 & 0.42 & 0.32 & 0.15 & 0.74 & 0.36 \\
\hline Work pressure and pace & 0.38 & 0.26 & 0.24 & 0.19 & 0.37 & 0.21 \\
\hline Staff training & 0.61 & 0.44 & 0.28 & 0.18 & 0.56 & 0.32 \\
\hline Office processes \& standardization & 0.65 & 0.57 & 0.31 & 0.25 & 0.56 & 0.39 \\
\hline Communication openness & 0.53 & 0.36 & 0.23 & 0.13 & 0.57 & 0.35 \\
\hline Patient care tracking & 0.63 & 0.28 & 0.44 & 0.23 & 0.60 & 0.19 \\
\hline Communication about errors & 0.64 & 0.42 & 0.26 & 0.14 & 0.65 & 0.40 \\
\hline Owner support for safety & 0.61 & 0.44 & 0.28 & 0.18 & 0.55 & 0.32 \\
\hline Organizational learning & 0.75 & 0.54 & 0.30 & 0.17 & 0.64 & 0.35 \\
\hline
\end{tabular}

$\mathrm{N}=934$ medical offices; Controls included region, owner type, number of providers, and practice type; $\mathrm{p}<.001$ for all variables

\section{Discussion}


We found full support for our hypotheses, with all organizational elements of patient safety climate having significant associations with all three perceived patient safety and quality outcomes. Therefore, it seems advantageous for medical offices to make an effort to improve upon all the different composites in the MOSOPS survey.

When examining its influence on all quality outcomes tested, the office processes and standardization composite appears to have the most consistent influence on perceived safety outcomes. Organizations that demonstrate evidence-based processes that include standardization are those that have a climate of safety [20]. Disorganization, lack of standardization, and inefficient processes are all targets of Lean methodology for process improvement that has impacted safety and quality [21-22].

Organizational learning had the second strongest relationship between overall perceptions of patient safety and quality, and the overall quality and patient safety rating. It had a moderate relationship with the frequency of patient safety and quality issues identified. Focusing on organizational learning through constantly monitoring and emphasizing clinical practices and outcomes can be essential to sustaining a healthy patient safety climate in outpatient medical facilities. A robust patient safety climate must include collaborative organizational learning [23].

A learning organization does an excellent job not only as it creates, acquires, and transfers knowledge, but also modifies behavior to reflect new knowledge. In our study we conceptualize organizational learning to be where processes are reviewed and changed based on recent evidence so the same mistakes don't happen again, and that there is validation that changes to processes worked. Healthcare organizations that do a better job with systematic organizational learning are more likely to have staff report medical errors, reports that can be used to improve overall patient safety [3].

Teamwork and communication about errors were other variables that had consistently strong positive associations with the perceived safety and quality outcomes, particularly overall perceptions and overall rating. When considering employees' perceptions of overall quality and safety from an organizational level, developing strong teams can make employees feel like they belong to a more receptive and cohesive organization. Levels of increased perceived organizational support led to more effective quality in their teams [24]. Improvements in inter-professional teamwork have been associated with similar care process improvements (Harris et al., 2016). In their study of primary care offices, Gehring et al. confirmed that the frequent use of team meetings involving all team members leads to strengthening the safety measures demonstrated by the team bringing about strategies and activities in error prevention [15]. Communication about errors reflects the ability and willingness to speak up when something isn't right. Hesitancy to speak up is a noted barrier to effective communication of errors, particularly among nurses [26]. The fear of reprisal and lack of impact of speaking up are two reasons that lead to an unwillingness to do so. Furthermore, the longstanding hierarchical norm between physicians and nurses leads to a role expectation among nurses that hinders their safety voice [27].

When we studied frequency of patient safety and quality issues, two different patient safety climate composites had the greatest influence, compared to when we studied 
overall perceptions and ratings: information exchange, and patient care tracking. It is possible that since the frequency measure is numeric and more concrete, it aligns more closely with more operational factors. Information exchange considers that when a medical office has problems exchanging accurate, complete, and timely information with external groups. It suggests that relationships with external agencies are important and can have a significant impact on the perception of quality of care provided. For example, discharge information sent from hospitals to medical offices is not deemed to sufficiently provide continuity of care [28]. Despite widespread adoption of electronic health records in medical offices, only a fraction shares data over information exchanges with external medical providers [29]. When there is information exchange there is a benefit to efficiency as well as safety. As an example, participation in health information exchanges, where data is shared in different delivery settings, has shown a reduction in repeat radiology imaging [30].

It is not surprising that patient care tracking has a strong relationship with the availability of medical records, medication problems, and other safety and quality issues. Patient care tracking emphasizes appropriate monitoring and follow up of patients and external providers regarding patient care. This is especially important in the outpatient setting, where primary care managers oversee overall care, but more specialized treatment is referred outside of the care manager's medical office. The biggest challenge with electronic health records remains the lack of interoperability among the systems [31].

\section{Limitations}

By the very nature of our data, only offices that elected to participate in the 2012 survey were included in our analysis, which could be a source of bias. For example, our sample had a higher percentage of Midwest medical offices and lower percentage of west offices, compared to nationwide. However, we had a high response rate $(71 \%$ average) within the participating medical offices so the results are representative of those participating medical offices. Offices that elected to participate in this survey may have a stronger emphasis on patient safety climate.

Because we used cross-sectional data on survey respondents, we are unable to infer causality from our analysis, and can merely make associations. Furthermore, we only had data that reflects perceptions of quality and safety outcomes, and do not have objective measures. Answers may reflect what respondents think is happening, but the reality may be different. However, research suggests there is a link between perceptions of safety climate and actual safety outcomes [12, 32].

Because our study was de-identified and cross-sectional, we were only able to associate certain elements of patient safety climate and perception with our DVs. Future research could include longitudinal work with baseline assessments of outpatient facilities, introduce interventions, and then re-assess the patient safety climate of the organization.

Although some limitations to the study exist, this research is a step towards understanding outpatient facilities, as it is a large sample of medical offices and 
respondents that can serve as a starting point for researchers interested in exploring specific elements of patient safety climate in a medical office setting.

\section{Conclusions}

Our findings are consistent with hospital research that indicates a positive relationship between positive elements of organizational patient safety climate and overall patient safety and quality perceptions, and the frequency of medical issues. Our results highlight the importance of all elements of safety climate, but in particular, developing standardized patient safety processes to potentially increase overall safety. Medical offices should consider targeting activities that improve upon those elements. Lean is a popular methodology that emphasizes standardization and reduction of wasteful processes. Although research on Lean in healthcare is still in its infancy, there is some evidence that it benefits process outcomes [33].

Our study also indicates that organizational learning is one of the elements most consistently associated of staff perceptions of patient safety and quality. To improve organizational learning, Chadwick and Raver suggest that organizations train leaders to support learning, encourage competitions that reward improvement in performance over time, and foster greater interdepartmental coordination to break down barriers to learning [34].

To encourage communication and a willingness to speak up, several approaches can be considered. Team-based care, where groups of medical professionals in different occupations manage groups of patients, can strengthen relationships along status lines [35]. Separately, organizations that use incident reporting systems should message them as learning systems and not punitive systems [36]. That will foster increased communication and a willingness to speak up.

We offer that not only hospitals but medical offices can have staff complete surveys to get an assessment of safety climate. Then those organizations can use results and make targeted improvements that may improve patient safety.

Author Contributions: Conceptualization, Jason P. Richter; Data curation, Jason P. Richter; Formal analysis, Matthew Calvert, Fernando Najera, Steven Wentz and Bob Wolfe; Funding acquisition, Forest Kim; Investigation, Matthew Calvert, Fernando Najera, Steven Wentz and Bob Wolfe; Methodology, Jason P. Richter; Project administration, Jason P. Richter and Forest Kim; Resources, Forest Kim; Software, Jason P. Richter and Forest Kim; Supervision, Jason P. Richter and Forest Kim; Validation, Jason P. Richter, Brad Beauvais, Lynn Downs and Forest Kim; Writing - original draft, Matthew Calvert, Fernando Najera, Steven Wentz and Bob Wolfe; Writing - review \& editing, Jason P. Richter, Brad Beauvais and Lynn Downs.

Funding: No external funding was required.

Institutional Review Board Statement: Not applicable

Informed Consent Statement: Not applicable

Data Availability Statement: Publicly available datasets were analyzed in this study. This data can be found here: https://www.ahrq.gov/sops/surveys/medical-office/index.html

Acknowledgments: We would like to thank the Agency for Healthcare Research and Quality for access to the data used in this study, and the Healthcare Research and Educational Trust for facilitating data access. 
Conflicts of Interest: The authors declare no conflict of interest

\section{References}

1. Sorra, J.; Nieva, V. Psychometric analysis of the hospital survey on patient safety. Final Report to Agency for Healthcare Research and Quality (AHRQ). Washington: AHRQ 2003.

2. Sorra, J.; Gray, L.; Famolaro, T.; Yount, N.; Behm, J. AHRQ Medical Office Survey on Patient Safety Culture: User's Guide. Prepared by Westat, under Contract No. HHSA290201300003C). AHRQ Publication 2016, 16.

3. Richter, J.P.; McAlearney, A.S.; Pennell, M.L. Evaluating the effect of safety culture on error reporting: a comparison of managerial and staff perspectives. American Journal of Medical Quality 2015, 30, 550-558.

4. Liu, C.; Liu, W.; Wang, Y.; Zhang, Z.; Wang, P. Patient safety culture in China: a case study in an outpatient setting in Beijing. BMJ quality $\mathcal{E}$ safety 2014, 23, 556-564.

5. Kuriakose, R.; Aggarwal, A.; Sohi, R.K.; Goel, R.; Rashmi, N.; Gambhir, R.S. Patient safety in primary and outpatient health care. Journal of family medicine and primary care 2020, 9,7 .

6. Gandhi, T.K.; Lee, T.H. Patient safety beyond the hospital. The New England journal of medicine 2010, 363, 1001.

7. Kaplan, M. The time has come to improve safety in ambulatory care. Available online: http://www.ihi.org/communities/blogs/the-time-hascome-to-improve-safety-in-ambulatory-care (accessed on December 28).

8. Stock, G.N.; McFadden, K.L. Improving service operations: linking safety culture to hospital performance. Journal of Service Management 2017.

9. Hoffmann, B.; Miessner, C.; Albay, Z.; Schröber, J.; Weppler, K.; Gerlach, F.M.; Güthlin, C. Impact of individual and team features of patient safety climate: a survey in family practices. The Annals of Family Medicine 2013, 11, 355-362.

10. Vogus, T.J.; Sutcliffe, K.M.; Weick, K.E. Doing no harm: enabling, enacting, and elaborating a culture of safety in health care. Academy of Management Perspectives 2010, 24, 60-77.

11. McFadden, K.L.; Stock, G.N.; Gowen, C.R., 3rd. Leadership, safety climate, and continuous quality improvement: impact on process quality and patient safety. Health Care Manage Rev 2015, 40, 24-34, doi:10.1097/HMR.0000000000000006.

12. Mardon, R.E.; Khanna, K.; Sorra, J.; Dyer, N.; Famolaro, T. Exploring relationships between hospital patient safety culture and adverse events. Journal of patient safety 2010, 6, 226-232.

13. Hofmann, D.A.; Mark, B. An investigation of the relationship between safety climate and medication errors as well as other nurse and patient outcomes. Personnel Psychology 2006, 59, 847-869.

14. Lee, S.-H.; Phan, P.H.; Dorman, T.; Weaver, S.J.; Pronovost, P.J. Handoffs, safety culture, and practices: evidence from the hospital survey on patient safety culture. BMC health services research 2016, 16, 1-8.

15. Gehring, K.; Schwappach, D.L.; Battaglia, M.; Buff, R.; Huber, F.; Sauter, P.; Wieser, M. Safety climate and its association with office type and team involvement in primary care. International journal for quality in health care 2013, 25, 394-402.

16. Carroll, R.J.; Ruppert, D. Transformation and weighting in regression; CRC Press: 1988; Volume 30.

17. Fan, C.J.; Pawlik, T.M.; Daniels, T.; Vernon, N.; Banks, K.; Westby, P.; Wick, E.C.; Sexton, J.B.; Makary, M.A. Association of safety culture with surgical site infection outcomes. Journal of the American College of Surgeons 2016, 222, 122-128.

18. Meddings, J.; Reichert, H.; Greene, M.T.; Safdar, N.; Krein, S.L.; Olmsted, R.N.; Watson, S.R.; Edson, B.; Lesher, M.A.; Saint, S. Evaluation of the association between Hospital Survey on Patient Safety Culture (HSOPS) measures and catheter-associated infections: results of two national collaboratives. BMJ quality \& safety 2017, 26, 226-235.

19. Fuller, C.M.; Simmering, M.J.; Atinc, G.; Atinc, Y.; Babin, B.J. Common methods variance detection in business research. Journal of Business Research 2016, 69, 3192-3198, doi:10.1016/j.jbusres.2015.12.008.

20. Sammer, C.E.; Lykens, K.; Singh, K.P.; Mains, D.A.; Lackan, N.A. What is patient safety culture? A review of the literature. Journal of Nursing Scholarship 2010, 42, 156-165.

21. Holden, R.J. Lean thinking in emergency departments: a critical review. Annals of emergency medicine 2011, 57, $265-278$. 
22. Smith, C.; Wood, S.; Beauvais, B. Thinking lean: implementing DMAIC methods to improve efficiency within a cystic fibrosis clinic. Journal for Healthcare Quality 2011, 33, 37-46.

23. Goh, S.C.; Chan, C.; Kuziemsky, C. Teamwork, organizational learning, patient safety and job outcomes. International journal of health care quality assurance 2013.

24. Howes, J.C.; Cropanzano, R.; Grandey, A.A.; Mohler, C.J. Who is supporting whom?: Quality team effectiveness and perceived organizational support. Journal of Quality Management 2000, 5, 207-223.

25. Harris, M.F.; Advocat, J.; Crabtree, B.F.; Levesque, J.-F.; Miller, W.L.; Gunn, J.M.; Hogg, W.; Scott, C.M.; Chase, S.M.; Halma, L. Interprofessional teamwork innovations for primary health care practices and practitioners: evidence from a comparison of reform in three countries. Journal of multidisciplinary healthcare 2016, 9, 35.

26. Okuyama, A.; Wagner, C.; Bijnen, B. Speaking up for patient safety by hospital-based health care professionals: a literature review. BMC health services research 2014, 14, 1-8.

27. Morrow, K.J.; Gustavson, A.M.; Jones, J. Speaking up behaviours (safety voices) of healthcare workers: A metasynthesis of qualitative research studies. International Journal of nursing studies 2016, 64, 42-51.

28. Flink, M.; Glas, S.B.; Airosa, F.; Öhlén, G.; Barach, P.; Hansagi, H.; Brommels, M.; Olsson, M. Patient-centered handovers between hospital and primary health care: an assessment of medical records. International journal of medical informatics 2015, 84, 355-362.

29. Furukawa, M.F.; King, J.; Patel, V.; Hsiao, C.-J.; Adler-Milstein, J.; Jha, A.K. Despite substantial progress in EHR adoption, health information exchange and patient engagement remain low in office settings. Health Affairs 2014, 33, 1672-1679.

30. Lammers, E.J.; Adler-Milstein, J.; Kocher, K.E. Does health information exchange reduce redundant imaging? Evidence from emergency departments. Medical care 2014, 227-234.

31. Rathert, C.; Porter, T.H.; Mittler, J.N.; Fleig-Palmer, M. Seven years after Meaningful Use: Physicians' and nurses' experiences with electronic health records. Health care management review 2019, 44, 30-40.

32. Cormier, D.; Gordon, I.M.; Magnan, M. Corporate environmental disclosure: contrasting management's perceptions with reality. Journal of Business Ethics 2004, 49, 143-165.

33. Moraros, J.; Lemstra, M.; Nwankwo, C. Lean interventions in healthcare: do they actually work? A systematic literature review. International Journal for Quality in Health Care 2016, 28, 150-165.

34. Chadwick, I.C.; Raver, J.L. Motivating organizations to learn: Goal orientation and its influence on organizational learning. Journal of management 2015, 41, 957-986.

35. Sheridan, B.; Chien, A.T.; Peters, A.S.; Rosenthal, M.B.; Brooks, J.V.; Singer, S.J. Team-based primary care: The medical assistant perspective. Health care management review 2018, 43, 115-125.

36. Hewitt, T.; Chreim, S.; Forster, A. Sociocultural factors influencing incident reporting among physicians and nurses: understanding frames underlying self-and peer-reporting practices. Journal of patient safety 2017, 13, 129-137. 